\title{
An ice crystal model for Jupiter's moon Europa
}

\author{
Karen Guldbæ к SCHMIDT, Dorthe DAHL-JENSEN \\ Center for Planetary Science, University of Copenhagen, Juliane Maries Vej 30, DK-2100 Copenhagen, Denmark \\ E-mail:kgs@gfy.ku.dk
}

\begin{abstract}
A simple model for crystal growth in the ice shell of Europa has been made in order to estimate the size of ice crystals at Europa's surface. If mass is lost from the surface of Europa due to sputtering processes, and the ice thickness is constant in time, ice crystals will be transported upwards in the ice shell. The crystals will therefore grow under varying conditions through the shell. The model predicts that ice crystals are $4 \mathrm{~cm}-$ $80 \mathrm{~m}$ across at the surface. For the preferred parameter values, a crystal size of the order of $7 \mathrm{~m}$ is calculated.
\end{abstract}

\section{INTRODUGTION}

Images of Jupiter's moon Europa from spacecraft show a bright young surface crisscrossed with linear structures, indicating an active environment. It is believed that Europa is covered by a water ice shell above an approximately $100 \mathrm{~km}$ deep ocean of liquid water (Andersen and others, 1998; Pappalardo and others, 1999).

The energy needed to keep the ocean from freezing under the cold surface conditions on Europa and for driving tectonic activity is believed to be dissipation energy from tidal forces acting on the ice shell. Europa is in synchronous rotation around Jupiter and should not experience time-varying deformation if the orbit is circular. However, an eccentric orbit is maintained by the Laplace resonance between the three inner Galilean satellites: Io, Europa and Ganymede. This causes the ice shell to flex $35 \mathrm{~m}$ with a period of 3.55 days (Greenberg and others, 1998).

The amount of tidal energy dissipated depends on the deformation properties of the ice shell, which are governed by ice thickness, temperature profile, ice crystal sizes, fabric and structure, and impurity content of the ice. These parameters have not been measured directly, so modelling, based on the data measured by spacecraft, is the only currently available way of gaining insight into possible processes and conditions in Europa's ice and ocean.

The idea of this study is to estimate the sizes of ice crystals at Europa's surface. It was inspired by looking at the Galileo images from the Conamara Chaos region, a region of disrupted surface, where polygonal blocks of ice up to $20 \mathrm{~km}$ in diameter have floated in a low-viscous layer below. Ice on Earth has a hexagonal crystal structure and is classified as ice $I_{h}$. This gives rise to the question: are the polygonal shapes of the ice blocks caused by the hexagonal structure of large ice crystals? It is plausible that large crystals could be found, because, unless disturbed, any crystal will continue to grow in time.

\section{AN ICE GRYSTAL MODEL}

Mass is lost from the surface of Europa due to sputtering by energetic ions (Ip and others, 1998). If the ice thickness at a given position is assumed to be constant in time, there should be freezing of sea water to the lower boundary of the ice, to compensate for the mass lost from the surface. An element of ice would therefore move upwards, relative to the boundaries of the ice, with a velocity corresponding to the rate of mass loss. As new ice crystals formed at the base of the ice shell are transported upwards, the ice temperature changes, which influences crystal growth. The crystals grow fast near the bottom, where the ice is at the melting point.

Ice crystals grow by grain boundary migration, a process mainly driven by the curvature of grain boundaries and by differences in stored energy between the grains. In this study, it is assumed that crystals grow following the normal grain-growth law (Paterson, 1994)

$$
D^{2}-D_{0}^{2}=k t,
$$

where $D^{2}$ is the cross-sectional area of crystals of age $t$ and $D_{0}^{2}$ is the initial crystal size. The growth rate $k$ is temperature-dependent and given by

$$
k=k_{0} \exp \left(-\frac{E}{R T}\right),
$$

where $E$ is the activation energy for grain boundary self-diffusion, $T$ is the temperature and $R$ is the gas constant.

For varying temperature through the ice, Equation (1) can be rewritten as

$$
\frac{\mathrm{d}\left(D^{2}\right)}{\mathrm{d} t}=k(T(t))
$$

where $\mathrm{d}\left(D^{2}\right)$ is the cross-sectional area by which the crystals grow.

For the ice crystal model for Europa, with $v=\mathrm{d} z / \mathrm{d} t$, the analytical expression for the crystal size at the surface is

$$
D_{\mathrm{s}}^{2}=\int_{-b}^{0} \frac{k}{v} \mathrm{~d} z=\int_{-b}^{0} \frac{k_{0}}{v} \exp \left(-\frac{E}{R T(z)}\right) \mathrm{d} z .
$$

The temperature profile $T(z)$ of the ice is determined by the volumetric tidal heat dissipation $q$, the temperature at the surface $T_{\mathrm{s}}$ and at the base $T_{\mathrm{b}}$. The temperature profile 
Table 1. Values of the parameters used

\begin{tabular}{ccc}
\hline Parameter & Ref.point & Range \\
\hline$v\left(\mathrm{~m} \mathrm{Myr}^{-1}\right)$ & 0.2 & $0.01-27$ \\
$b(\mathrm{~km})$ & 2.0 & $0.2-10$ \\
$T_{\mathrm{b}}(\mathrm{K})$ & 273 & $240-273$ \\
$T_{\mathrm{s}}(\mathrm{K})$ & 100 & $50-130$ \\
\hline
\end{tabular}

for the ice shell used in this study is given by (Chyba and others, 1998)

$$
T(z)=T_{\mathrm{s}} \exp \left(\frac{z}{h}\right)
$$

where $T_{\mathrm{s}}$ is the temperature at the surface and $h=b \ln \left(T_{\mathrm{b}} / T_{\mathrm{S}}\right)$.

Equations (4) and (5) are solved using a finite-difference scheme, and the final crystal size at the surface $D_{\mathrm{s}}^{2}$ is found. The sizes of the crystals are described by a leng th parameter $d_{\mathrm{s}}$ corresponding to the diameter of a circle of area $D_{\mathrm{s}}^{2}$. In order to calculate $d_{\mathrm{s}}$ the values of the following parameters are needed: the ice thickness $b$, the mass loss rate due to sputtering $v$, the surface temperature $T_{\mathrm{s}}$, and the base temperature $T_{\mathrm{b}}$.

For each parameter a reference value is chosen based on spacecraft measurements and models. Since there is an uncertainty in these values, tests are made for a range of values around the reference value. In these tests, the surface crystal size $d_{\mathrm{S}}$ is found for the range of values listed in Table 1 for the examined parameter, and the rest of the parameters held constant at their reference values.

The age of the crystals at the surface is the total time it takes for them to be moved from base to surface. The initial crystal size is assumed to be $d_{0}=10^{-8} \mathrm{~m}$. The model is not sensitive to this value, since the crystal typically grows several $\mathrm{cm}$ or tens of $\mathrm{cm}$ in the first iteration.

For the reference values, an ice shell with a net mass-loss rate of $v=0.2 \mathrm{~m} \mathrm{Myr}^{-1}$, an ice thickness $b=2 \mathrm{~km}$, a surface temperature $T_{\mathrm{s}}=100 \mathrm{~K}$, and a base temperature $T_{\mathrm{b}}=$ $273 \mathrm{~K}$, a crystal size in the upper part of the ice of $d_{\mathrm{s}}=7 \mathrm{~m}$ is found (Fig. 1).
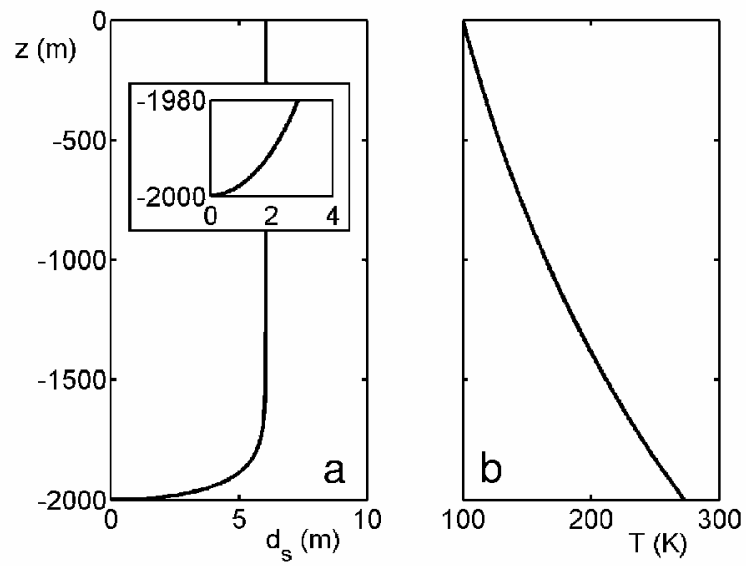

Fig. 1. (a) Plot of crystal size $d(z)$ vs depth $z$ for an ice shell with thickness $b=2000 \mathrm{~m}$ and a mass-loss rate $v=$ $0.2 \mathrm{~m} \mathrm{Myr}^{-1}$; inset is a close-up of the lowermost $20 \mathrm{~m}$. (b) Plot of temperature $T(z)$ vs depth $z$. The temperatures at the boundaries are $T_{\mathrm{s}}=100 \mathrm{~K}$ and $T_{\mathrm{b}}=273 \mathrm{~K}$.

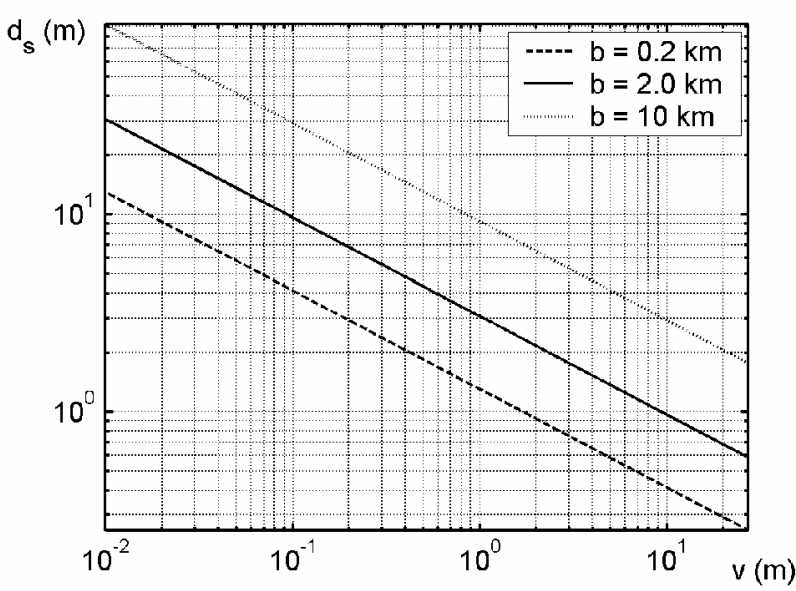

Fig. 2. The crystal size at the surface $d_{\mathrm{s}}$ as a function of rate of mass loss $v$ for three different ice thicknesses.

\section{DISGUSSION AND RESULTS FOR THE VALUES OF PARAMETERS USED}

\section{Rate of mass loss}

Mass loss due to sputtering has been detected at the surface of Europa. The rate of mass loss estimated by Ip and others (1998) is $v=0.2 \mathrm{~m} \mathrm{Myr}^{-1}$. Cooper and others (2001) gave an estimate $v=0.016 \mathrm{~m} \mathrm{Myr}^{-1}$ of the lower limit for the rate of mass loss.

The rate of mass loss can also be constrained by a simple calculation of how long it takes to erase details in the surface topography. A typical vertical scale of the topography for the stratigraphically oldest plain areas on Europa is $10 \mathrm{~m}$ (Pappalardo and others, 1999). Larger topography variations also occur; an example is the rim-to-floor depth of $\sim 300 \mathrm{~m}$ for the crater Govannan (Moore and others, 1998). This vertical scale of topography should be erased on the time-scale of the age of the surface. The surface age, found by crater counts, has been estimated to be $55 \mathrm{Myr}$ with an uncertainty of a factor of 5 (Zahnle and others, 1998, with corrections by Pappalardo and others, 1999). The limits for the rate of mass loss thus become: $v_{\min }=10 \mathrm{~m} /$ $(55 \times 5) \mathrm{Myr}=0.04 \mathrm{~m} \mathrm{Myr}^{-1}$ and $v_{\max }=300 \mathrm{~m} /(55 / 5) \mathrm{Myr}$ $=27 \mathrm{~m} \mathrm{Myr}^{-1}$.

A range of values for the mass-loss rate $v$ of $0.01-$ $27 \mathrm{~m} \mathrm{Myr}^{-1}$ are used for the calculation of $d_{\mathrm{s}}$. In Figure 2, $d_{\mathrm{S}}$ as a function of $v$ for different ice thicknesses is shown. The crystal size is inversely proportional to the rate of mass loss. Larger ice crystals are produced for low values of $v$. This is because a low mass-loss rate $v$ causes a slow vertical ice velocity in the ice shell, resulting in longer travel times for the ice crystals through the ice shell.

\section{Ice thickness}

From modelling of Europa's gravitational field, it is estimated that the outermost $80-170 \mathrm{~km}$ consists of water in the form of ice or liquid (Anderson and others, 1998). The thickness of the ice shell is estimated by theoretical modelling and geological observations. A thin ice thickness makes a liquid ocean probable.

The thickness of the ice shell on top of the liquid ocean is controlled by energy balance in the system. The tidal heat dissipation depends on the ice thickness, since a thin ice is easier to deform than a thick ice. The phenomena of dissipa- 

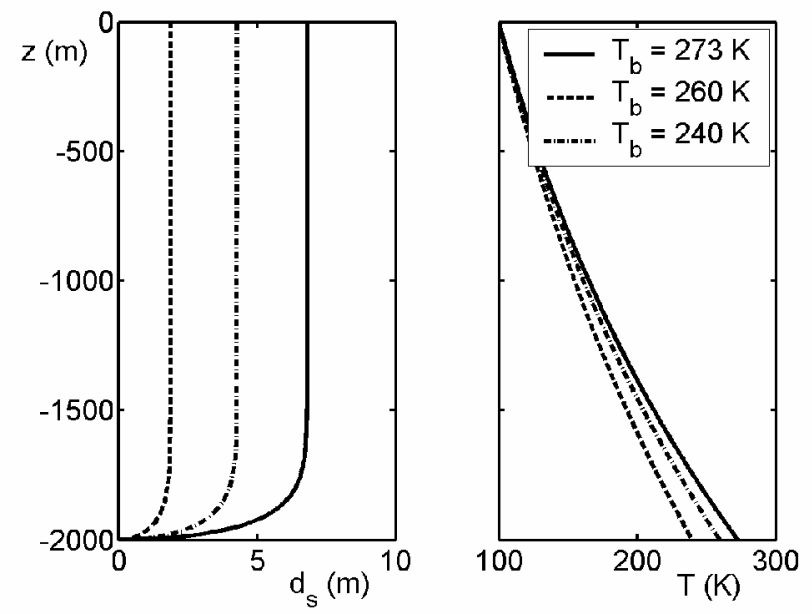

Fig. 3. Calculated crystal sizes for three different base temperatures.

tive heating and cooling by conduction or convection involve non-linear feedback mechanisms. They are associated with the dependence of temperature on internal heat production from deformation and heat flow into the ice at its boundaries, as well as the dependence of deformation properties of the ice on temperature.

There are presently differing opinions on the thickness of the ice shell. In the thick-ice model the ice shell is composed of a warm convecting layer of ice beneath a cold, thin rigid lid. There may be a liquid ocean under the convecting layer. In the thin-ice model the ice shell is just a few $\mathrm{km}$ thick, and it lies on top of a liquid ocean. For the thick-ice model, ice thicknesses are usually estimated to be $5-25 \mathrm{~km}$, where the rigid lid is assumed to be a few $\mathrm{km}$ thick (Pappalardo and others, 1999). For the thin-ice model, ice thicknesses are usually estimated to be $0.2-3.0 \mathrm{~km}$ (Williams and Greeley, 1998).

Both the thin-ice and the thick-ice model are adaptable with the crystal growth model. For the thin-ice model, the solution is straightforward: the thickness of the ice shell corresponds to the ice thickness $b$ used in the crystal model. For the thick-ice model, the thickness of the rigid lid is used as the ice thickness in the model, as this layer is the crystalline heat-conducting part of the ice, where the model set-up can be applied.

A range of values of the ice thickness of $0.2-10 \mathrm{~km}$ was used for calculation of $d_{\mathrm{s}}$.

A thick ice shell causes the crystals to travel for a longer time through the shell, so that they grow for a correspondingly longer period than for a thin ice shell. As seen in Figure 2, for large values of the ice thickness $b$, large values of $d_{\mathrm{s}}$ are found.

\section{Temperature at the ice-ocean intersection}

The salt concentration of the ocean and total ice thickness of the ice shell are unknown, so a range of base temperatures seem reasonable to test, since it is most probable that the freezing-point temperature at the ice-ocean intersection is $<273 \mathrm{~K}$. An important reason for this is that soluble salts lower the freezing-point temperature of the water by several tens of ${ }^{\circ} \mathrm{C}$. Another reason is that the pressure of the overlying ice shell decreases the freezing-point temperature of the sea water by a few ${ }^{\circ} \mathrm{C}$. This is a second-order effect compared to the effect of the salt.
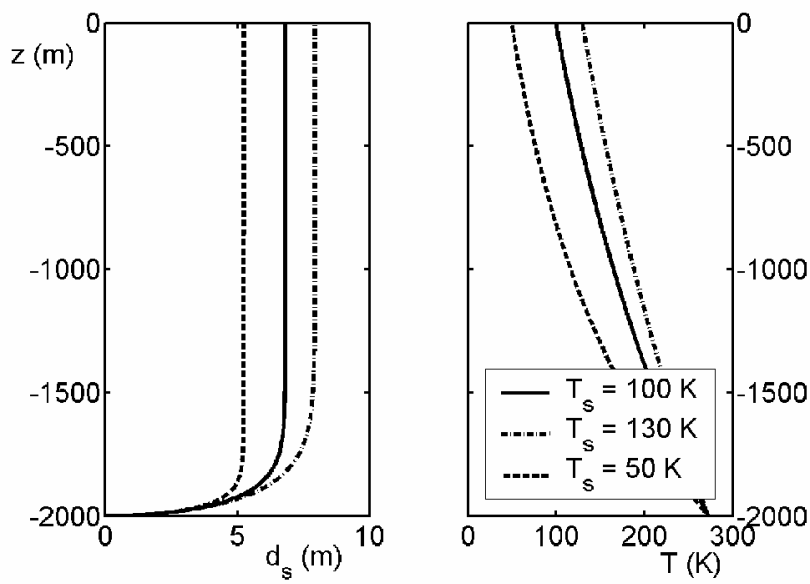

\section{Fig. 4. Calculated crystal sizes for three different surface tem- peratures.}

For the thin-ice model, the base temperature of Europa's ice shell must be at the freezing-point temperature of the sea water beneath. For the thick-ice model, the base temperature is the adiabatic temperature of the convecting layer underneath, which is $T_{\mathrm{ad}}=260 \mathrm{~K}$ (McKinnon, 1999).

A range of base temperatures from $240-273 \mathrm{~K}$ are used in calculations of $d_{\mathrm{s}}$.

Figure 3 shows the influence of $T_{\mathrm{b}}$ on $d_{\mathrm{s}}$. It is seen that $d_{\mathrm{s}}$ increases for increasing values of $T_{\mathrm{b}}$, as the crystals grow with a larger rate in a warmer ice.

There may be large spatial variations in the ice thickness due to spatial variations in tidal deformation, surface temperature variations with latitude, and uneven distribution of heat from the core. Convection and subsequent mixing of the sea water may, however, even out the latter effect, as well as result in an even distribution of salt concentration in the sea water.

As the melting-point temperature at the base of thick ice is lower than that of thin ice due to the increased pressure, this would result in slower grain growth in the thick ice. Therefore, competing processes are present in this case. For the thick ice, crystal growth would be slower. On the other hand, the larger ice thickness would give the crystals a longer time to grow.

\section{Surface temperature}

Values of the surface temperature $T_{\mathrm{S}}$ calculated from the black-body radiation equilibrium are found in the range 50$110 \mathrm{~K}$, with an area-averaged mean of $100 \mathrm{~K}$ (Ojakangas and Stevenson, 1989). Measurements from the Galileo spacecraft are in agreement with this, as the measurements yield values for low-latitude diurnal brightness temperatures in the range 86-132 K (Spencer and others, 1999).

Surface temperatures in the range $50-130 \mathrm{~K}$ are used in calculations of grain growth.

The crystal diameter $d_{\mathrm{S}}$ increases with increasing values of the surface temperature $T_{\mathrm{S}}$ as the crystals will grow at a larger rate in a warmer ice (Fig. 4).

\section{DISGUSSION}

The broad range of the parameters used results in surface crystal sizes from $d_{\min }=4 \mathrm{~cm}$ to $d_{\max }=80 \mathrm{~m}$. It has been estimated from surface spectra that the crystal sizes are 50- 
$100 \mu \mathrm{m}$ at the surface (Shirley and Carlson, 2000). However, sputtering redistributes material at a rate of $0.8 \mathrm{~m} \mathrm{Myr}^{-1}$ (Ip and others, 1998), and a thin upper layer of small-grained redistributed material may exist above possible large-grained old ice.

A change in crystal diameter of about $1 \mathrm{~m}$ occurs for a variation in the surface temperature of a few tens of ${ }^{\circ} \mathrm{C}$. The same change in crystal diameter occurs for a variation in the base temperature of only a few ${ }^{\circ} \mathrm{C}$. The reason for this is that the change of shape of the temperature profile by changing the surface temperature occurs in the upper region of the ice shell, where crystal growth is not as effective as it is near the base.

Lake Vostok on the Antarctic ice sheet has often been used as an analogy to the physical settings on Europa, since it is a large lake under $4 \mathrm{~km}$ of ice (Jouzel and others, 1999). Above Lake Vostok, a deep ice core has been drilled to $120 \mathrm{~m}$ above the ice-lake intersection. The glacier ice above is estimated to be 420000 years old, and the lower $90 \mathrm{~m}$ of ice consists of frozen lake water and is estimated to be roughly 1 Myr old (Petit and others, 1999). In this part of the ice core, ice crystals of sizes $>0.2-1.0 \mathrm{~m}$ have been found (Jouzel and others, 1999). The large ice crystals from Vostok show that large crystals are indeed a possibility when the crystals have a long time to grow.

The impurity content of the ice on Europa is a very uncertain parameter, which influences the crystal growth rate $k$. At the surface, the relative concentration of non-ice and ice-rich components has been measured (McCord and others, 1998). This provides information on what chemical species may be expected, but their quantities are hard to deduce. Sputtering processes are likely to alter the chemical composition at the surface, so the composition of the ice below the surface may differ from this.

For the process of crystal growth, the content of soluble impurities in the ice is important, since it may impede crystal growth (Paterson, 1991). However, the salt content in the ice would be less than that of the sea water, since part of the salt is expelled during freezing. The amount of salt expelled is controlled by the freezing rate of the ice.

The stresses in the ice shell are not included in the calculations, where crystal sizes are calculated on the basis of normal grain growth. The effect of the stresses may be that a fabric develops which makes deformation easier in some directions than others. A fabric may also be developed during formation of the ice crystals, as seen in sea ice on Earth. Bending stresses in the ice shell may lead to polygonization. However, the presence of cracks in the ice shell must absorb part of the deformation, so strains are decreased compared to an unfractured shell.

In order to determine how the different mechanisms may influence crystal growth, a stress-dependent growth law is needed. The fabric would influence the deformation properties of the ice, so an anisotropic flow law would have to be used to calculate the tidal heating. In case the crystal sizes influence the flow properties of the ice as suggested by Goldsby and Kohlstedt (1997), this would also have to be included in the calculations.

In the crystal growth model, the travel time of the crystals through the ice shell is equivalent to the age of the crystals when they reach the surface, and can be calculated to be $t_{\text {ice }}=b / v$, where $b$ is the total ice thickness and $v$ is massloss rate. For an ice thickness of $2000 \mathrm{~m}$ and a mass-loss rate of $0.2 \mathrm{~m} \mathrm{Myr}^{-1}$, this yields $t_{\text {ice }}=2000 \mathrm{~m} / 0.2 \mathrm{~m} \mathrm{Myr}^{-1}=$
$10 \mathrm{Gyr}$. This age is more than the age of the solar system and Europa. However, the initial freezing of the ice where the ice shell was built up must have taken place at a faster rate than the present rate of freezing. This would cause the age- - depth relation to be non-linear, and the upper crystals to be younger than the values calculated as in the previous example. The freezing of the ice from the water must always have taken place at the base of the ice, where it is warm. So all the crystals will have experienced a period of high temperature and fast growth rates.

On Earth it is known that the size and orientation of ice crystals on the surface of the ice sheets and at the base of sea ice influence the scattering of radar (Zwally, 1977; Tucker and others, 1992). This is caused by the dielectric contrast between ice grains and their surroundings (e.g. air or impurities at grain boundaries). Thus it cannot be ruled out that the ice crystal sizes affect radar scattering on Europa.

\section{GONGLUSION}

Model calculations predicts that there are large ice crystals in Europa's ice shell, $4 \mathrm{~cm}-80 \mathrm{~m}$ across, with a value of $7 \mathrm{~m}$ for the preferred parameter values.

The ice crystal sizes are important for the deformation and fracture properties of the ice shell, and will therefore affect the tidal heat dissipation.

The calculated large sizes of ice crystals make it more plausible that the polygonal shapes of the ice blocks are caused by the lattice structure of large ice crystals. The blocks of ice may have separated along weak zones such as crystal boundaries.

As radar backscatter may be influenced by the crystal sizes, it will be important to know these in order to design an orbiting radar sounder, which is a possible method for detecting an ocean under the ice shell on Europa.

\section{AGKNOWLEDGEMENT}

This work was supported by the FREJA programme "Life of ice - ice in the planetary system" funded by the Danish Natural Science Research Council.

\section{REFERENGES}

Anderson, J. D., G. Schubert, R. A. Jacobson, E. L. Lau, W. B. Moore and W. L. Sjogren. 1998. Europa's differentiated internal structure: inferences from four Galileo encounters. Science, 281(5385), 2019-2022.

Chyba, C. F., S. J. Ostro and B.C. Edwards. 1998. Radar detectability of a subsurface ocean on Europa. Icarus, 134(2), 292-302.

Cooper, J. F., R. E. Johnson, B. H. Mauk, H. B. Garrett and N. Gehrels. 2001. Energetic ion and electron irradiation of the icy Galilean satellites. Icarus, 149(1), 133-159.

Goldsby, D. L. and D. L. Kohlstedt. 1997. Grain boundary sliding in finegrained ice I. Scripta Mater., 37(9), 1399-1406.

Greenberg, R. and 9 others. 1998. Tectonic processes on Europa: tidal stresses, mechanical response, and visible features. Icarus, 135(1), 64-78.

Ip, W.-H., D. J. Williams, R.W. McEntire and B. H. Mauk. 1998. Ion sputtering and surface erosion at Europa. Geophys. Res. Lett., 25(6), 829-832.

Jouzel, J. and 9 others. 1999. More than $200 \mathrm{~m}$ of lake ice above subglacial Lake Vostok, Antarctica. Science, 286(5447), 2138-2141.

McCord, T. B. and 12 others. 1998. Salts on Europa's surface detected by Galileo's near infrared mapping spectrometer. Science, 280(5367), 1242-1245.

McKinnon, W. B. 1999. Convective instability in Europa's floating ice shell. Geophys. Res. Lett., 26(7), 951-954.

Moore, J. M. and 17 others. 1998. Large impact features on Europa: results of the Galileo nominal mission. Icarus, 135(1), 127-145.

Ojakangas, G. W. and D. J. Stevenson. 1989. Thermal state of an ice shell on Europa. Icarus, 81 (2), 220-241. 
Pappalardo, R. T. and 21 others. 1999. Does Europa have a subsurface ocean? Evaluation of the geological evidence. F. Geophys. Res., 104(E10), 24,015-24,055.

Paterson, W. S. B. 1991. Why ice-ageice is sometimes "soft". Cold Reg. Sci. Technol., 20(1), 75-98.

Paterson, W. S. B. 1994. The physics of glaciers. Thirdedition. Oxford, etc., Elsevier.

Petit, J.-R. and 18 others. 1999. Climate and atmospheric history of the past 420,000 years from the Vostok ice core, Antarctica. Nature, 399(6735), 429-436.

Shirley, J. H. and R.W. Carlson. 2000. Water ice grain size variations on Europe. [Abstract.] Eos, 81(48), Fall Meeting Supplement, F793.

Spencer, J. R., L. K. Tamppari, T. Z. Martin and L. D. Travis. 1999. Temperatures on Europa from Galileo photopolarimeter-radiometer: night- time thermal anomalies. Science, 284(5419), 1514-1516.

Tucker, W. B., III, D. K. Perovich, A. J. Gow, W. F. Weeks and M. R. Drinkwater. 1992. Physical properties of sea ice relevant to remote sensing. In Carsey, F. D. and 7 others, eds. Microwave remote sensing of sea ice. Washington, DC, American Geophysical Union, 9-28. (Geophysical Monograph Series 68 .

Williams, K. K. and R. Greeley. 1998. Estimates of ice thickness in the Conamara Chaos region of Europa. Geophys. Res. Lett., 25(23), 4273-4276.

Zahnle, K., L. Dones and H. F. Levison. 1998. Cratering rates on the Galilean satellites. Icarus, 136(2), 202-222

Zwally, H.J. 1977. Microwave emissivity and accumulation rate of polar firn. F. Glaciol., 18(79), 195-215. 